Check for updates

Cite this: J. Mater. Chem. B, 2018, 6, 8064

Received 2nd October 2018, Accepted 6th November 2018 DOI: $10.1039 / c 8 t b 02598 c$

rsc.li/materials-b

\title{
Bioinspired reversible hydrogel adhesives for wet and underwater surfaces $\dagger$
}

\author{
Hoon Yi, ${ }^{a}$ Sung Ho Lee, ${ }^{b}$ Minho Seong, ${ }^{a}$ Moon Kyu Kwak (D) ${ }^{b}$ and \\ Hoon Eui Jeong $(\mathbb{D} * a$
}

\begin{abstract}
Stable and reversible adhesion to wet surfaces is challenging owing to water molecules at the contact interface. In this study, we develop a hydrogel-based wet adhesive, which can exhibit strong and reversible adhesion to wet and underwater surfaces as well as to dry surfaces. The remarkable wet adhesion of the hydrogel adhesive is realized based on a synergetic integration of bioinspired microarchitectures and water-friendly and water-absorbing properties of the polymeric hydrogel. Under dry conditions, the microstructured hydrogel adhesive exhibits strong van der Waals interaction-based adhesion, while under underwater conditions, it can maximize capillary adhesion. Consequently, the hydrogel adhesive exhibits remarkable adhesion strengths for dry, moist, and submerged substrates. Maximum normal and shear adhesion strengths of 423 and 384, 492 and 340, and 253 and $21 \mathrm{kPa}$ are achieved with the hydrogel adhesive for dry, moist, and submerged substrates, respectively. Our results demonstrate that strong wet and underwater adhesion can be achieved only with the hydrogel-based adhesive with simple microscale architecture.
\end{abstract}

\section{Introduction}

Robust adhesion to wet surfaces is of significance in diverse applications such as medical operations, marine structures, energy devices operating under wet environments (e.g., fuel cells), transfer printing, and even daily goods. ${ }^{1-8}$ Stable adhesion between surfaces can be obtained by keeping the distance between the surfaces very close to the atomic level. In typical dry environments, such tight contact between surfaces can be easily achieved by utilizing materials with low effective elastic moduli so that they can follow the roughness of the target surface. ${ }^{9-17}$ However, in wet environments, water molecules not only penetrate gaps between the surfaces easily, but also reduce the adhesion strengths of the adhesive materials by hydrating and decomposing them. Consequently, it is very challenging to ensure good adhesion under wet conditions ${ }^{1,3,8,18-20}$ Although cyanoacrylate (Super Glue ${ }^{\mathrm{TM}}$ ) is a strong tissue adhesive, it is cytotoxic, and incompatible with wet or underwater surfaces because the uncured adhesive rapidly solidifies into a glassy phase when it comes into contact with water. ${ }^{21,22}$ In order to overcome such difficulties, synthetic wet adhesives emulating

\footnotetext{
${ }^{a}$ Department of Mechanical Engineering, Ulsan National Institute of Science and Technology, Ulsan 689-798, Republic of Korea. E-mail: hoonejeong@unist.ac.kr

${ }^{b}$ Department of Mechanical Engineering, Kyungpook National University, Daegu 702-701, Republic of Korea

$\dagger$ Electronic supplementary information (ESI) available. See DOI: 10.1039/ c8tb02598c
}

natural adhesive materials of marine organisms have been extensively investigated. ${ }^{23}$ Representative examples include wet adhesives mimicking the proteins present in biological system such as mussel adhesives, ${ }^{23-26}$ barnacle cements, ${ }^{27}$ and sandcastle-worm glues. ${ }^{28}$ These marine organism-inspired synthetic wet adhesives typically have the use of 3,4-dihydroxyphenylalanine (DOPA) in common, and many different types of DOPA-based wet adhesives have demonstrated effective wet adhesion performance. ${ }^{1,5,7,29}$ However, they also pose several limitations such as adhesion degradation by wet-air oxidation, long contact-forming time, and high production cost. In addition, they are not suitable for applications that require reversible and reusable wet adhesion, as they are typically permanent adhesives that lack reversible adhesion capability ${ }^{1,30}$

Bioinspired dry adhesives based on micro- or nanostructure arrays that emulate biological fibrillar adhesives have emerged as reversible and reusable adhesives for various dry surfaces. Their reversible dry adhesion could be realized by maximizing van der Waals interactions with the target surface with the aid of the micro- or nanostructure array. ${ }^{31-41}$ Most of the existing bioinspired dry adhesives are composed of elastomers with hydrophobic surface properties (e.g., polydimethylsiloxane (PDMS)). ${ }^{42-49}$ This indicates that they have potential as wet or underwater adhesives because hydrophobic elastomers promote removal of the water at the contact interface and therefore initiate interfacial adhesion in wet environments. ${ }^{50,51}$ In reality, however, water entrapment often occurs at the contact interface even when using a hydrophobic elastomer because it is difficult 
to perfectly avoid or remove any entrapped water across the entire surface of elastomeric samples of macroscopic size. ${ }^{52}$ In this case, the entrapped water acts as a flaw for initiating debonding at the interface, ${ }^{52}$ resulting in limited wet adhesion performance. ${ }^{1,3,8,18-20}$ In this regard, hydrogels have immense potential as reversible wet adhesives when they are integrated with biomimetic microstructures, based on the following factors. First, the hydrophilic nature of the hydrogels is beneficial to induce strong capillary adhesion under wet conditions. Second, they can absorb a large quantity of water at the contact interface, which would allow closer contact with the target substrate. $^{3,19,53-55}$ Furthermore, they are biocompatible and therefore can be used for diverse biomedical applications such as tissue adhesives, tissue repair, and wound dressings. Indeed, in recent years, hydrogels have been actively explored as materials for efficient wet adhesives. ${ }^{21,22,52,56}$ Engineered tough hydrogel adhesives have been demonstrated to have remarkable adhesion performance with an adhesion energy over $1000 \mathrm{~J} \mathrm{~m}^{-2}$ to various substrates including skin, tissue, glass, and metals. ${ }^{21,22,56}$ However, they also have several shortcomings such as requirements for specific functional groups, long contact-forming time, and irreversible adhesion. ${ }^{21,22,52,56}$ To address the shortcomings, hydrogel adhesives with unique micro- and nano-structures have been used, and their fast and reversible underwater adhesion performance has been demonstrated based on ionic and hydrogen bonds, mechanical interlocking, and $\mathrm{pH}$-sensitive swelling. ${ }^{3,52,57}$ Nonetheless, the research toward reversible, fast, and strong adhesives for wet and underwater surfaces is still in its infancy and requires further development. ${ }^{52}$

In this study, we develop a bioinspired wet adhesive utilizing a hydrogel, which can exhibit immediate, strong and reversible adhesion under wet and underwater conditions based on the water-absorbing properties and capillary adhesion of the microstructured hydrogel. The hydrogel adhesive consists of a bioinspired micropillar array made of the biocompatible polyethylene glycol (PEG) hydrogel, which can absorb a large quantity of water and is hydrophilic. In this PEG-based bioinspired adhesive, the biomimetic microarchitecture enables conformal physical contact to target surfaces, while the PEG polymer absorbs water at the contact interface and enables capillary adhesion. Consequently, robust and reversible wet adhesion can be achieved under wet and underwater conditions only with the hydrogel adhesive, without the need for complex surface modification with chemical moieties. The wet adhesion behavior of the bioinspired hydrogel adhesive well agrees with theoretical models.

\section{Experimental methods}

\section{Fabrication of the hydrogel adhesive}

A master mold with a microhole array was prepared by photolithography using a bilayer of a lift-off resist (LOR, Microchem Corporation) and SU-8 photoresist. First, the LOR was spincoated onto a Si wafer followed by baking at $200{ }^{\circ} \mathrm{C}$ for $10 \mathrm{~min}$.
SU-8 was then spin-coated onto the LOR layer and baked at $100{ }^{\circ} \mathrm{C}$ for $5 \mathrm{~min}$. Subsequently, the SU-8/LOR bilayer on the wafer was exposed to ultraviolet (UV) light $(\lambda=365 \mathrm{~nm}$, dose = $\left.250 \mathrm{~mJ} \mathrm{~cm}^{-2}\right)$, followed by post-exposure baking $\left(100{ }^{\circ} \mathrm{C}\right.$ for $3 \mathrm{~min}$ ). Microhole array patterns were then generated in the SU-8 layer by development using a SU-8 developer. Subsequently, selective removal of the LOR layer was carried out to form an undercut in the LOR layer using a LOR developer (AZ 400K, AZ Electronics Materials Corporation). In order to generate a PEG dimethacrylate (PEGDMA) adhesive, a drop of an $80 \mathrm{wt} \%$ PEGDMA solution (molecular weight: 750, Sigma Aldrich) mixed with $20 \mathrm{wt} \%$ of deionized (DI) water and $0.2 \mathrm{wt} \%$ of an Irgacure 2959 photoinitiator (Sigma Aldrich) was uniformly dispensed onto the patterned master. ${ }^{58-61}$ Subsequent photopolymerization of the PEGDMA solution by UV irradiation $\left(\lambda=365 \mathrm{~nm}\right.$, dose $\left.=300 \mathrm{~mJ} \mathrm{~cm}^{-2}\right)$ and removal of the cured PEGDMA from the master yielded the PEGDMA adhesive with a bioinspired microstructure array. ${ }^{62,63}$

\section{Surface characterization}

Scanning electron microscopy (SEM) images of the fabricated PEGDMA sample were acquired using a HITACHI S-4800 microscope (Hitachi, Japan). To image the swollen PEGDMA microstructures, the PEGDMA sample was first submerged in DI water for $12 \mathrm{~h}$. Next, the swollen PEGDMA sample was lyophilized. In order to avoid charging effects, the PEGDMA sample was coated with a $5 \mathrm{~nm}$-thick Pt layer using a sputter coater (K575X, Quorum Emitech, UK). The water contact angles (CAs) on the planar PEGDMA and glass substrates were measured using a drop shape analyzer (SDLAB 200TEZD, FEMTOFAB, Korea) at room temperature $\left(25{ }^{\circ} \mathrm{C}\right)$. The measurements for each sample were performed five times and the average values were used.

\section{Adhesion measurements}

The normal and shear adhesion of the adhesive samples were measured with custom-built equipment. The equipment consisted of motorized movable parts along the vertical and horizontal directions, load cells connected to the movable parts, and the sample holder. Adhesive samples with sizes of $1 \pm$ $0.01 \mathrm{~cm}^{2}$ were attached to dry, moist, and submerged substrates (glass, $\mathrm{Au}$, and porcine skin) with controlled preloads in the range of 20-300 $\mathrm{kPa}$. After the application of the preload, the motorized part was moved along the vertical (normal adhesion test) or horizontal (shear adhesion test) axis with a retraction rate of $0.5 \mathrm{~mm} \mathrm{~s}^{-1}$ until the occurrence of separation at the adhesive/substrate interface. For the adhesion measurements against the moist substrate, a $3 \mu \mathrm{L}$ water droplet was uniformly dispensed on the substrate to an area of $1 \times 1 \mathrm{~cm}^{2}$. Subsequently, preloads were applied to the samples for a few seconds before the measurement. For the underwater adhesion measurements, the substrates were affixed to a mount in a small water bath placed on the adhesion test equipment. The adhesive samples were then submerged in the water bath for $10 \mathrm{~min}$, followed by the pull-off and shear strength measurements. For each experimental condition, two specimens 
were tested and ten repeated adhesion measurements were conducted for each specimen. The averaged values and standard deviations of the measurements were used for the adhesion data.

\section{Results and discussion}

\section{Fabrication and structure of the hydrogel adhesive}

Fig. 1a shows a schematic of the hydrogel adhesive fabrication. As described above, the hydrogel adhesive was prepared using a replica molding technique against a patterned master using PEGDMA as a base material. PEGDMA is a hydrophilic polymeric hydrogel, which can absorb a large quantity of water. It can be rapidly cured by UV light irradiation within a few tens of seconds, which enables precise and scalable fabrication of diverse micro/nanostructures. In addition, its mechanical properties can be easily modulated by adjusting its concentration in water. Furthermore, it is biocompatible and thus can be utilized for various biomedical applications. ${ }^{3,19}$ Fig. 1b shows the fabricated PEGDMA hydrogel adhesive. The resulting PEGDMA adhesive is in the form of a highly flexible film on which a uniform microstructure array is regularly formed (Fig. 1b-i). The individual microstructures have protruding heads on cylindrical stems (Fig. 1b-ii and iii). The protruding heads of the microstructures are beneficial to obtain high adhesion strengths against various surfaces based on van der Waals interactions without any surface treatment with adhesive chemical moieties. According to previous studies, the adhesion strengths of the microstructures with protruding heads are 5-26 times higher than those of simple microstructures with flat or hemispherical ends. ${ }^{34}$ The fabricated microstructures had a tip diameter of $24 \mu \mathrm{m}$, stem diameter of $20 \mu \mathrm{m}$, height of $25 \mu \mathrm{m}$, and spacing of $20 \mu \mathrm{m}$. It is worth noting that the fabricated PEGDMA in this study does not have a supporting layer (e.g., polyethylene terephthalate), as it leads to macroscopic bending of the adhesive film in the presence of water due to a mismatch in volume expansion between the swollen PEGDMA layer and nonswellable supporting layer. ${ }^{19}$
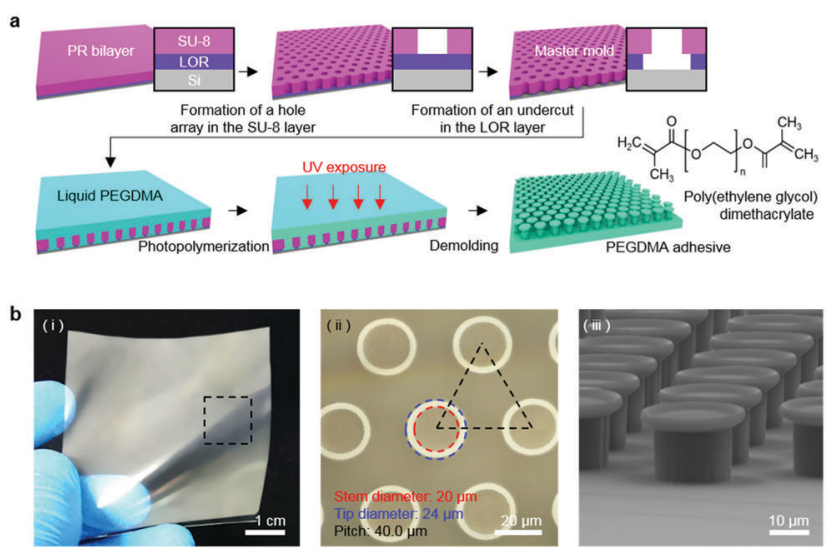

Fig. 1 (a) Schematic of the fabrication procedure of the PEGDMA adhesive. (b) (i) Photograph, (ii) microscopy image, and (iii) SEM image of the fabricated PEGDMA adhesive.

\section{Wet adhesion behavior of the hydrogel adhesive}

In order to evaluate the adhesion properties of the PEGDMA adhesive, we quantitatively measured the pull-off and shear strengths of the adhesive against a flat glass substrate under dry, wet, and underwater conditions (Fig. 2a). Under the wet conditions, the adhesion measurements were performed against a glass surface moistened with water. Under the underwater conditions, the adhesion measurements were performed against a submerged substrate. Adhesion of a polydimethylsiloxane (PDMS) adhesive was also evaluated for comparison. Fig. 2b-i and ii show the measured pull-off and shear strengths of the PDMS and PEGDMA adhesives with a preload of $20 \mathrm{kPa}$ for the three different conditions. Overall, under the dry conditions, the PDMS and PEGDMA adhesives exhibited similar adhesion at a preload of $20 \mathrm{kPa}$. The normal adhesion strengths of the PDMS and PEGDMA adhesives were 152.3 and $160.6 \mathrm{kPa}$, respectively (Fig. 2c-i). The shear strengths of the PDMS and PEGDMA adhesives were 143.8 and $153.2 \mathrm{kPa}$, respectively (Fig. 2c-ii). However, for the moist substrate, the PDMS and PEGDMA adhesives exhibited completely different adhesion behaviors. The pull-off (normal) and shear strengths of the PDMS adhesive were significantly reduced to 40.2 and $24.2 \mathrm{kPa}$, respectively, which are $26.4 \%$ and $16.8 \%$, respectively, of those against the dry substrate. This indicates that the PDMS adhesive has limited utility as a wet adhesive. On the contrary, the pull-off strength of the PEGDMA adhesive maintained a rather high value (143.4 $\mathrm{kPa})$ against the wet substrate (Fig. 2c-i),

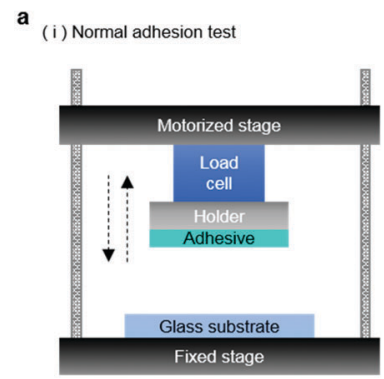

(iii ) Shear adhesion test

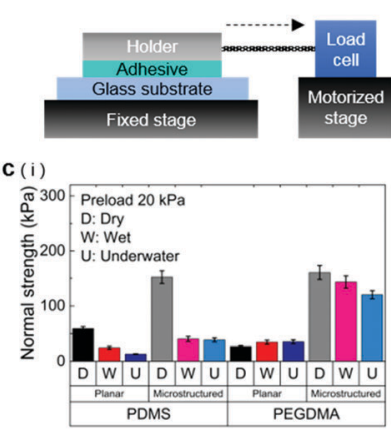

b
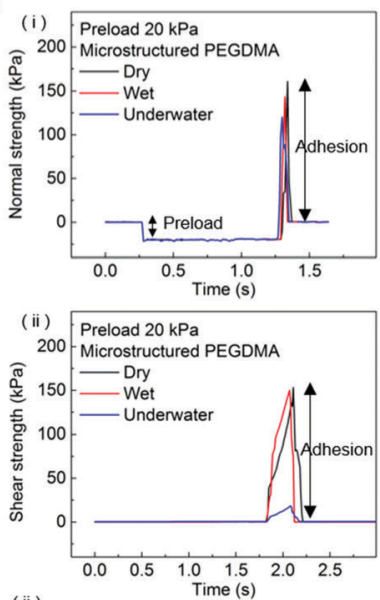

(ii)

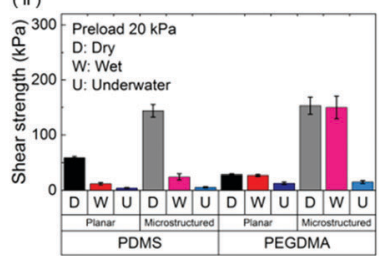

Fig. 2 (a) Experimental setups for (i) normal and (ii) shear adhesion measurements. (b) Representative examples of the measured (i) normal and (ii) shear adhesion under the three different conditions at a preload of $20 \mathrm{kPa}$. (c) (i) Normal and (ii) shear adhesion strengths of the PEGDMA and PDMS adhesives under the three different conditions at a preload of $20 \mathrm{kPa}$. Error bars represent standard deviations $(N=20)$. 
which is equal to $89.3 \%$ of that for the dry substrate. In addition to the normal adhesion, the shear strength of the PEGDMA adhesive also remained at a high level (150 kPa) (Fig. 2c-ii), which is $97.9 \%$ of that for the dry substrate. The PEGDMA adhesive exhibited significant adhesion even to the submerged substrate (Fig. 2b and c). For the submerged substrate, the PEGDMA adhesive exhibited a normal adhesion strength of $120.2 \mathrm{kPa}$, whereas the PDMS adhesive exhibited a significantly degraded normal adhesion strength of $38.5 \mathrm{kPa}$. The shear adhesion of the PEGDMA adhesive decreased to $15.4 \mathrm{kPa}$ for the submerged surface. However, this value is 2.75 times higher than that of the PDMS adhesive $(5.6 \mathrm{kPa})$.

In order to further evaluate the wet adhesion performance of the PEGDMA adhesive, its normal and shear adhesion strengths were investigated at different preloads in the range of 20-300 kPa (Fig. 3). Under dry conditions (Fig. 4a-i), the maximum normal and shear adhesion strengths of the PEGDMA adhesive were 422.9 and $384.2 \mathrm{kPa}$ at a preload of $300 \mathrm{kPa}$, respectively (Fig. 3a and b). In contrast, the maximum normal and shear adhesion strengths of the PDMS adhesive were $185.6 \mathrm{kPa}$ (at a preload of $100 \mathrm{kPa}$ ) and $170.5 \mathrm{kPa}$ (at a preload
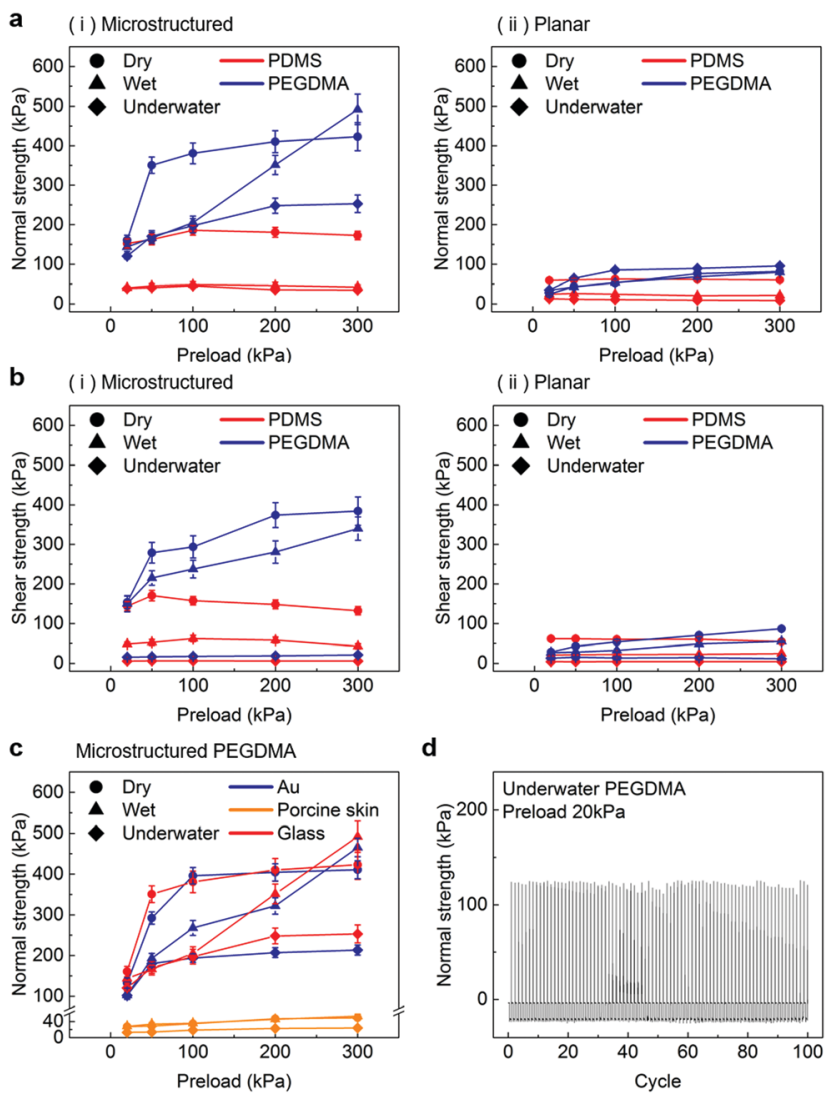

Fig. 3 (a) Normal and (b) shear adhesion strengths of the (i) microstructured and (ii) planar adhesives under the different conditions as a function of the preload. (c) Normal adhesion strengths of the microstructured PEGDMA adhesives on different substrates, namely glass, Au, and porcine skin. (d) Repeatability test results showing the normal adhesion strength of the PEGDMA adhesive under underwater conditions (preload: $20 \mathrm{kPa}$ ). Error bars represent standard deviations (for (a)-(c); $N=20$ ). of $50 \mathrm{kPa}$ ), respectively. Theoretically, the normal and shear adhesion forces of microstructures with protruding tips are: ${ }^{64,65}$

$$
\begin{aligned}
& P_{\text {normal }}=A \sqrt{\frac{\pi W_{12} E}{2 a\left(1-\nu^{2}\right)}} \\
& P_{\text {shear }}=\mu A \sqrt{\frac{\pi W_{12} E}{2 a\left(1-\nu^{2}\right)}}
\end{aligned}
$$

where $A$ is the contact area, $W_{12}$ is the work of adhesion between the contacting surfaces, $E$ is the elastic modulus of the material used for the microstructures, $\nu$ is the Poisson's ratio of the material, $a$ is the radius of the detached zone of the microstructures with protruding tips, and $\mu$ is the friction coefficient. The higher surface energy $\left(42.5 \mathrm{~mJ} \mathrm{~m}^{-2}\right)$ and elastic modulus (15.5 MPa) of PEGDMA than those of PDMS (surface energy: $20.5 \mathrm{~mJ} \mathrm{~m}^{-2}$, elastic modulus: $1-2 \mathrm{MPa}$ ) lead to higher adhesion to the dry surface. ${ }^{19,43,66,67}$ Our experimental adhesiontest results for the dry substrate are in agreement with the theoretical predictions $(\mu \approx 1.0$, as shown in Fig. 4e; see Fig. S1 (ESI $\dagger$ ) for the determination of the friction coefficient). It is worth noting that the relatively higher adhesion of the PEGDMA adhesive compared with that of the PDMS adhesive could be attributed to the hydrogen bonds between the PEG hydrogel and glass substrate. ${ }^{68}$ To test this hypothesis, we performed additional adhesion measurements against $\mathrm{Au}$ and skin substrates (Fig. 3c and Fig. S3, ESI $\dagger$ ). According to the measurement results, the PEGDMA adhesives exhibit overall similar adhesion strengths on the Au substrate compared to those on the glass substrate. It is observed that the adhesion strength of the planar PDMS on the glass was higher than that of the planar PEGDMA sample on the glass (Fig. 2c). These results indicate that the hydrogen bonds between the PEGDMA adhesive and glass do not play a critical role in the measured macroscopic adhesion of the microstructured PEGDMA adhesive. The adhesion strengths of the PEGDMA adhesive on skin were much lower than those on the glass or Au substrates due to the relatively high surface roughness of skin compared with that of the flat glass or Au substrates.

For a moist substrate, the PEGDMA adhesive exhibited maximum normal and shear strengths of 491.8 and $340 \mathrm{kPa}$ at a preload of $300 \mathrm{kPa}$, respectively, which are similar to those of the PEGDMA adhesive for the dry substrate (Fig. 3a and b). On the contrary, for the moist substrate, the maximum normal and shear strengths of the PDMS adhesive were significantly reduced to 48.3 and $31.5 \mathrm{kPa}$ (at a preload of $100 \mathrm{kPa}$ ), respectively (Fig. 3a and b). The remarkable adhesion strengths of the PEGDMA adhesive for the moist substrate mainly originated from the swelling behavior of the PEGDMA hydrogel, which can absorb a large amount of water. When the microstructured PEGDMA adhesive contacts the water on the substrate, the water is entrapped in the three-dimensional polymeric network of the PEGDMA microstructures (Fig. 4b), leading to partial or complete removal of the water on the substrate (Fig. 4a-ii). Accordingly, the PEGDMA adhesive can be in closer contact with the substrate. Furthermore, the diameter of the hydrogel 


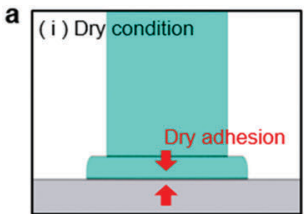

b

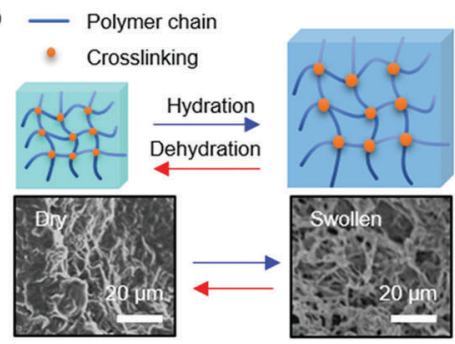

$\mathbf{e}_{\text {(i) Dry normal strength }}$

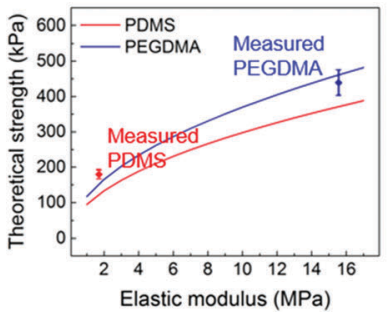

(ii) ) Dry shear strength

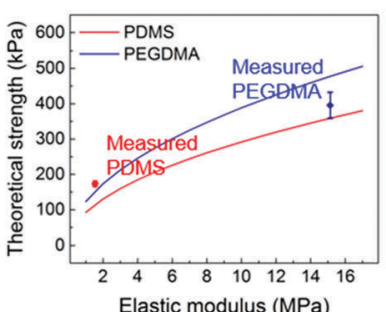

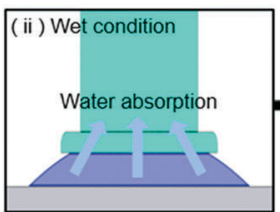

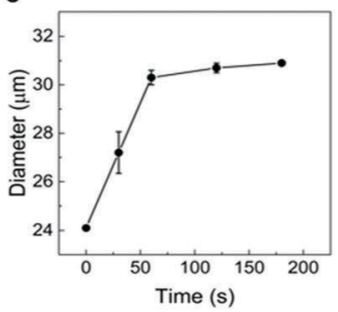

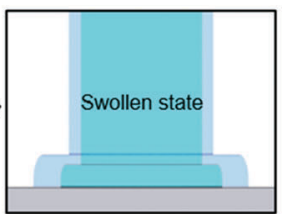

d
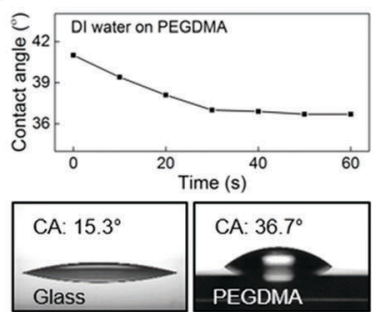

f (i) Wet normal strength

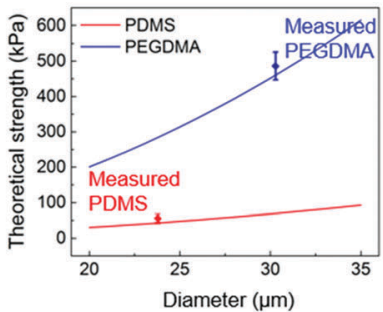

(ii) Wet shear strength

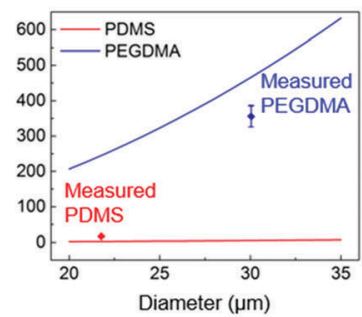

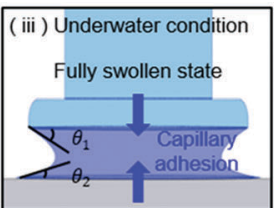

g (i) Underwater normal strength

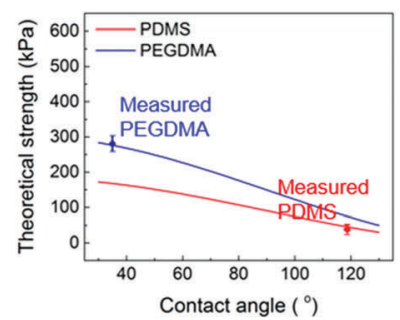

(ii) Underwater shear strength

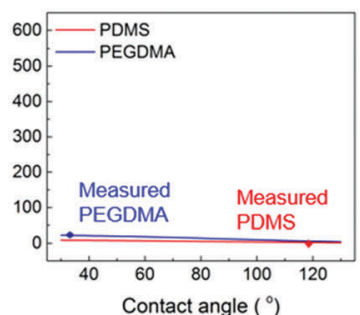

Fig. 4 (a) Conceptual illustrations showing the PEGDMA adhesive in contact with (i) dry, (ii) moist, and (iii) submerged substrates. (b) Conceptual illustration of the reversible swelling behavior of the PEGDMA hydrogel. (c) Diameter of the PEGDMA microstructures as a function of the water exposure time. (d) Water CA on the planar PEGDMA surface as a function of the time. Comparisons of the measured (i) normal and (ii) shear adhesion with the theoretical predictions for the (e) dry, (f) wet, and (g) underwater conditions. Error bars represent standard deviations (for (e)-(g); $N=20)$.

microstructures increased owing to the swelling of the hydrogel (Fig. 4c). Consequently, a high level of adhesion can be obtained with the PEG-hydrogel-based adhesive for the moist substrate. The maximum experimental adhesion values for this case followed eqn (1) well (Fig. 4f). On the contrary, in the case of the PDMS adhesive, the water on the substrate could not be perfectly removed despite the hydrophobic nature of PDMS. Consequently, the water remaining on the substrate hinders close contact between the microstructure and substrate, leading to the remarkably reduced normal and shear adhesion strengths. ${ }^{1,6,8,18-20}$ In this case, the adhesion originated mainly from capillary adhesion. The capillary adhesion between two adjacent surfaces is expressed as: ${ }^{69,70}$

$$
P_{\text {capillary }}=\frac{\gamma \pi r^{2}}{h}\left(\cos \theta_{1}+\cos \theta_{2}\right)
$$

where $\gamma$ is the surface tension of water, $r$ is the contact radius, $h$ is the thickness of the water layer, and $\theta_{1}$ and $\theta_{2}$ are the water
CAs at the two surfaces. The adhesion behavior of the PDMS adhesive for the wet surface agrees well with eqn (2) (Fig. 4g).

The PEGDMA adhesive exhibited significant adhesion even to the submerged surface. The maximum normal adhesion of the PEGDMA adhesive reached $253 \mathrm{kPa}$ (at a preload of $300 \mathrm{kPa}$ ), whereas that of the PDMS adhesive was $42.2 \mathrm{kPa}$ (at a preload of $100 \mathrm{kPa}$ ). The superior underwater adhesion of the PEGDMA adhesive is based on enhanced capillary adhesion owing to the water-friendly characteristic of the PEGDMA hydrogel. According to eqn (2), the capillarity-induced normal adhesion strength is higher for a hydrophilic material with a lower water CA. In the case of PEGDMA, the water CA monotonously decreased with the increase in the water exposure time, reaching $36^{\circ}$ at equilibrium (Fig. 4d), while that of PDMS was approximately $120^{\circ}$ regardless of the water exposure time. The theoretically calculated adhesion strength of the PEGDMA adhesive is $274.8 \mathrm{kPa}$ for a submerged glass substrate, while that of the PDMS adhesive is $43.7 \mathrm{kPa}$, which agrees well with our experimental observations (Fig. 4g). The maximum shear adhesion 
strength of the PEGDMA adhesive under the underwater conditions was $21.2 \mathrm{kPa}$, significantly lower than the maximum normal adhesion value. The reduced shear strength for the underwater surface can be explained as stable friction cannot be established under the wet conditions owing to the water layer, which obstructs the direct solid-solid contact. The friction coefficients of waterlubricated PDMS and PEGDMA were estimated to be $\sim 0.05$ and 0.08, respectively (Fig. S1, ESI $\dagger$ ). Accordingly, the underwater shear strength $\left(P_{\text {shear }}=\mu P_{\text {capillary }}\right)$ is significantly reduced compared to the underwater normal strength. Nevertheless, the underwater shear strength of the PEGDMA adhesive $(21.2 \mathrm{kPa})$ is 3.4 times higher than that of the PDMS adhesive (6.24 $\mathrm{kPa})$. Furthermore, the PEGDMA adhesion was highly reversible. The high level of underwater adhesion could be maintained over repeated cycles of adhesion tests (Fig. 3d). For all the experimental conditions, the PEGDMA and PDMS adhesives with bioinspired microstructures exhibited significantly higher adhesion strengths than those of nonpatterned planar PEGDMA and PDMS samples (Fig. 3a-ii and b-ii). This indicates that the bioinspired microstructures of our adhesive have proper structural topographies as robust and reversible adhesives. Our adhesive is also highly flexible and is completely made of biocompatible PEG hydrogel. Therefore, it holds strong potential for applications in flexible and reversible skin-and tissue-attachable patches. Indeed, although the adhesion strength of the PEGDMA adhesives was lower on skin as compared with that on the flat glass or Au substrates, the skin adhesion of PEGDMA was in the range of $\sim 25-45 \mathrm{kPa}$, which is comparable to those of existing skin adhesives. ${ }^{17,71}$ Therefore, by incorporating emerging flexible electronics and functional chemical moieties for more stable skin and tissue adhesion, this PEGDMA adhesive can be used in flexible patch-type platforms for advanced biomedical or healthcare devices.

\section{Conclusions}

We demonstrated the high and reversible wet and underwater adhesion performance of a bioinspired PEG hydrogel adhesive. By simply combining the water-absorbing and swelling properties of the polymeric hydrogel and bioinspired microscale architectures, high normal and shear adhesion strengths could be obtained with the adhesive for moist and submerged substrates, which surpassed those of previously reported bioinspired reversible wet adhesives. In addition to that to the wet and underwater surfaces, our hydrogel adhesive exhibited remarkable adhesion to a dry surface. Therefore, our hydrogel adhesive represents a universal adhesive that can strongly and reversibly adhere to dry, moist, and submerged surfaces. This bioinspired hydrogel adhesive with smart adhesion performance could be used as a versatile and advanced adhesive for various biomedical applications that require stable and repeatable adhesion under diverse dry and wet environments.

\section{Conflicts of interest}

There are no conflicts to declare.

\section{Acknowledgements}

This work was supported by the Institute for Information \& communications Technology Promotion (IITP) grant funded by the Korea government (MSIP) (No. 2017000910001100, 20180007560011001).

\section{References}

1 Y. H. Zhao, Y. Wu, L. Wang, M. M. Zhang, X. Chen, M. J. Liu, J. Fan, J. Q. Liu, F. Zhou and Z. K. Wang, Nat. Commun., 2017, 8, 2218.

2 D. K. Wainwright, T. Kleinteich, A. Kleinteich, S. N. Gorb and A. P. Summers, Biol. Lett., 2013, 9, 20130234.

3 H. H. Park, M. Seong, K. Sun, H. Ko, S. M. Kim and H. E. Jeong, ACS Macro Lett., 2017, 6, 1325-1330.

4 Y. F. Ma, S. H. Ma, Y. Wu, X. W. Pei, S. N. Gorb, Z. K. Wang, W. M. Liu and F. Zhou, Adv. Mater., 2018, 30, 1801595.

5 J. Liu, Q. L. Yang, J. J. Xu, K. S. Liu, L. Guo and L. Jiang, Prog. Chem., 2012, 24, 1946-1954.

6 M. Li, J. Xie, Q. W. Dai, W. Huang and X. L. Wang, J. Mech. Behav. Biomed. Mater., 2018, 78, 266-272.

7 H. Lee, B. P. Lee and P. B. Messersmith, Nature, 2007, 448, 338-341.

8 H. Ko, M. Seong and H. E. Jeong, Soft Matter, 2017, 13, 8419-8425.

9 K. Autumn, C. Majidi, R. E. Groff, A. Dittmore and R. Fearing, J. Exp. Biol., 2006, 209, 3558-3568.

10 G. Carbone and E. Pierro, Small, 2012, 8, 1449-1454.

11 H. E. Jeong, J. K. Lee, H. N. Kim, S. H. Moon and K. Y. Suh, Proc. Natl. Acad. Sci. U. S. A., 2009, 106, 5639-5644.

12 J. Jeong, J. Kim, K. Song, K. Autumn and J. Lee, J. R. Soc., Interface, 2014, 11, 140627.

13 S. M. Kang, Int. J. Precis. Eng. Manuf. - Green Technol., 2016, 3, 189-192.

14 M. Rohrig, M. Thiel, M. Worgull and H. Holscher, Small, 2012, 8, 3009-3015.

15 D. P. Wang, A. W. Zhao, R. Jiang, D. Li, M. F. Zhang, Z. B. Gan, W. Y. Tao, H. Y. Guo and T. Mei, Appl. Surf. Sci., 2012, 259, 93-98.

16 M. Zhou, N. Pesika, H. B. Zeng, Y. Tian and J. Israelachvili, Friction, 2013, 1, 114-129.

17 I. Hwang, H. N. Kim, M. Seong, S. H. Lee, M. Kang, H. Yi, W. G. Bae, M. K. Kwak and H. E. Jeong, Adv. Healthcare Mater., 2018, 7, 1800275.

18 L. Heepe, A. E. Kovalev and S. N. Gorb, Beilstein J. Nanotechnol., 2014, 5, 903-909.

19 H. Yi, M. Seong, K. Sun, I. Hwang, K. Lee, C. Cha, T. I. Kim and H. E. Jeong, Adv. Funct. Mater., 2018, 28, 1706498.

20 A. Y. Stark, I. Badge, N. A. Wucinich, T. W. Sullivan, P. H. Niewiarowski and A. Dhinojwala, Proc. Natl. Acad. Sci. U. S. A., 2013, 110, 6340-6345.

21 J. W. Yang, R. B. Bai and Z. G. Suo, Adv. Mater., 2018, 30, 1800671.

22 J. Li, A. D. Celiz, J. Yang, Q. Yang, I. Wamala, W. Whyte, B. R. Seo, N. V. Vasilyev, J. J. Vlassak, Z. Suo and D. J. Mooney, Science, 2017, 357, 378-381. 
23 B. K. Ahn, J. Am. Chem. Soc., 2017, 139, 10166-10171.

24 Y. Xu, Q. H. Liu, A. Narayanan, D. Jain, A. Dhinojwala and A. Joy, Adv. Mater. Interfaces, 2017, 4, 1700506.

25 M. A. North, C. A. Del Grosso and J. J. Wilker, ACS Appl. Mater. Interfaces, 2017, 9, 7866-7872.

26 B. P. Lee, C. Y. Chao, F. N. Nunalee, E. Motan, K. R. Shull and P. B. Messersmith, Macromolecules, 2006, 39, 1740-1748.

27 S. K. Clancy, A. Sodano, D. J. Cunningham, S. S. Huang, P. J. Zalicki, S. Shin and B. K. Ahn, Biomacromolecules, 2016, 17, 1869-1874.

28 H. Shao and R. J. Stewart, Adv. Mater., 2010, 22, 729-733.

29 Y. C. Choi, J. S. Choi, Y. J. Jung and Y. W. Cho, J. Mater. Chem. B, 2014, 2, 201-209.

30 B. Soltannia and D. Sameoto, ACS Appl. Mater. Interfaces, 2014, 6, 21995-22003.

31 H. Jiang, E. W. Hawkes, C. Fuller, M. A. Estrada, S. A. Suresh, N. Abcouwer, A. K. Han, S. Q. Wang, C. J. Ploch, A. Parness and M. R. Cutkosky, Sci. Robot., 2017, 2, 4545.

32 H. Kasem, A. Tsipenyuk and M. Varenberg, Soft Matter, 2015, 11, 2909-2915.

33 M. K. Kwak, H. E. Jeong, W. G. Bae, H. S. Jung and K. Y. Suh, Small, 2011, 7, 2296-2300.

34 H. Shahsavan, S. M. Salili, A. Jakli and B. X. Zhao, Adv. Mater., 2017, 29, 1604021.

35 Y. Wang, H. Hu, J. Y. Shao and Y. C. Ding, ACS Appl. Mater. Interfaces, 2014, 6, 2213-2218.

36 W. Federle, J. Exp. Biol., 2006, 209, 2611-2621.

37 C. Greiner, E. Arzt and A. del Campo, Adv. Mater., 2009, 21, 0801548.

38 G. Huber, H. Mantz, R. Spolenak, K. Mecke, K. Jacobs, S. N. Gorb and E. Arzt, Proc. Natl. Acad. Sci. U. S. A., 2005, 102, 16293-16296.

39 S. Kim, M. Sitti, T. Xie and X. C. Xia, Soft Matter, 2009, 5, 3689-3693.

40 H. Ko, H. Yi and H. E. Jeong, Int. J. Precis. Eng. Manuf. - Green Technol., 2017, 4, 273-280.

41 P. C. Lin, S. Vajpayee, A. Jagota, C. Y. Hui and S. Yang, Soft Matter, 2008, 4, 1830-1835.

42 J. Purtov, M. Frensemeier and E. Kroner, ACS Appl. Mater. Interfaces, 2015, 7, 24127-24135.

43 H. Yi, M. Kang, M. K. Kwak and H. E. Jeong, ACS Appl. Mater. Interfaces, 2016, 8, 22671-22678.

44 D. Sameoto, H. Sharif and C. Menon, J. Adhes. Sci. Technol., 2012, 26, 2641-2652.

45 M. P. Murphy, B. Aksak and M. Sitti, Small, 2009, 5, 170-175.

46 Y. Menguc, S. Y. Yang, S. Kim, J. A. Rogers and M. Sitti, Adv. Funct. Mater., 2012, 22, 1246-1254.

47 S. H. Ma, D. A. Wang, Y. M. Liang, B. Q. Sun, S. N. Gorb and F. Zhou, Small, 2015, 11, 1131-1137.

48 N. W. Rizzo, K. H. Gardner, D. J. Walls, N. M. Keiper-Hrynko, T. S. Ganzke and D. L. Hallahan, J. R. Soc., Interface, 2006, 3, 441-451.
49 H. Yi, I. Hwang, M. Sung, D. Lee, J. H. Kim, S. M. Kang, W. G. Bae and H. E. Jeong, Int. J. Precis. Eng. Manuf. - Green Technol., 2014, 1, 347-351.

50 A. P. Defante, A. Nyarko, S. Kaur, T. N. Burai and A. Dhinojwala, Langmuir, 2018, 34, 4084-4094.

51 M. K. Chaudhury and G. M. Whitesides, Langmuir, 1991, 7, 1013-1025.

52 P. Rao, T. L. Sun, L. Chen, R. Takahashi, G. Shinohara, H. Guo, D. R. King, T. Kurokawa and J. P. Gong, Adv. Mater., 2018, 30, 1801884.

53 R. Kempaiah and Z. H. Nie, J. Mater. Chem. B, 2014, 2, 2357-2368.

54 K. Y. Seong, M. S. Seo, D. Y. Hwang, E. D. O'Cearbhaill, S. Sreenan, J. M. Karp and S. Y. Yang, J. Controlled Release, 2017, 265, 48-56.

55 S. Y. Yang, E. D. O’Cearbhaill, G. C. Sisk, K. M. Park, W. K. Cho, M. Villiger, B. E. Bouma, B. Pomahac and J. M. Karp, Nat. Commun., 2013, 4, 1702.

56 H. Yuk, T. Zhang, S. T. Lin, G. A. Parada and X. H. Zhao, Nat. Mater., 2016, 15, 190-198.

57 S. H. Ma, M. Scaraggi, P. Lin, B. Yu, D. A. Wang, D. Dini and F. Zhou, J. Phys. Chem. C, 2017, 121, 8452-8463.

58 H. C. Gu, B. F. Ye, H. B. Ding, C. H. Liu, Y. J. Zhao and Z. Z. Gu, J. Mater. Chem. C, 2015, 3, 6607-6612.

59 D. J. Guo, H. Zhang, J. B. Li, S. M. Fang, Z. D. Dai and W. Tan, J. Mater. Chem. B, 2013, 1, 379-386.

60 C. Heo, C. Jeong, H. S. Im, J. U. Kim, J. Woo, J. Y. Lee, B. Park, M. Suh and T. I. Kim, Nanoscale, 2017, 9, 17743-17751.

61 J. H. Lee, Y. M. Lee, Y. H. Kim, J. Y. Lee, S. J. Choi and P. J. Yoo, Small, 2011, 7, 2587-2592.

62 M. K. Kwak, H. E. Jeong, T. I. Kim, H. Yoon and K. Y. Suh, Soft Matter, 2010, 6, 1849-1857.

63 S. H. Lee, S. W. Kim, C. W. Park, H. E. Jeong, J. G. Ok and M. K. Kwak, Int. J. Precis. Eng. Manuf. - Green Technol., 2017, 4, 177-181.

64 G. Carbone, E. Pierro and S. N. Gorb, Soft Matter, 2011, 7, 5545-5552.

65 Y. Tian, N. Pesika, H. B. Zeng, K. Rosenberg, B. X. Zhao, P. McGuiggan, K. Autumn and J. Israelachvili, Proc. Natl. Acad. Sci. U. S. A., 2006, 103, 19320-19325.

66 E. S. Pane, J. E. A. Palamara and H. H. Messer, Dent. Mater., 2012, 28, E150-E159.

67 H. Seong, K. Pak, M. Joo, J. Choi and S. G. Im, Adv. Electron. Mater., 2016, 2, 1500209.

68 A. H. Hofman, I. A. van Hees, J. Yang and M. Kamperman, Adv. Mater., 2018, 30, 1704640.

69 E. Cheung and M. Sitti, J. Adhes. Sci. Technol., 2008, 22, 569-589.

70 H. Peisker, L. Heepe, A. E. Kovalev and S. N. Gorb, J. R. Soc., Interface, 2014, 11, 140752.

71 S. Baik, D. W. Kim, Y. Park, T. J. Lee, S. H. Bhang and C. Pang, Nature, 2017, 546, 396-400. 